\title{
Structural alterations of the intestinal epithelial barrier in Parkinson's disease
}

\author{
Thomas Clairembault 1,2,3† ${ }^{1}$ Laurène Leclair-Visonneau ${ }^{1,2,4+}$, Emmanuel Coron ${ }^{1,2,3,4}$, Arnaud Bourreille ${ }^{1,3,4}$, Séverine Le Dily ${ }^{4}$, \\ Fabienne Vavasseur ${ }^{3,4}$, Marie-Françoise Heymann ${ }^{2,5,6}$, Michel Neunlist ${ }^{1,2,3}$ and Pascal Derkinderen ${ }^{1,2,4,7^{*}}$
}

\begin{abstract}
Functional and morphological alterations of the intestinal epithelial barrier (IEB) have been consistently reported in digestive disorders such as irritable bowel syndrome and inflammatory bowel disease. There is mounting evidence that Parkinson's disease (PD) is not only a brain disease but also a digestive disorder. Gastrointestinal involvement is a frequent and early event in the course of PD, and it may be critically involved in the early development of the disease. We therefore undertook the present survey to investigate whether changes in the IEB function and/or morphology occur in PD. Colonic biopsies were performed in 31 PD patients and 11 age-matched healthy controls. The para- and transcellular permeability were evaluated by measuring sulfonic acid and horseradish peroxidase flux respectively, in colonic biopsies mounted in Ussing chambers. The expression and localization of the two tight junctions proteins ZO-1 and occludin were analyzed by Western blot and immunofluorescence, respectively. The para- and transcellular permeability were not different between PD patients and controls. The expression of occludin, but not ZO-1, was significantly lower in colonic samples from PD patients as compared to controls and the cellular distribution of both proteins was altered in colonic mucosal specimens from PD patients. Our findings provide evidence that the IEB is morphologically altered in PD and further reinforce the potential role of the gastrointestinal tract in the initiation and/or the progression of the disease.
\end{abstract}

Keywords: Parkinson's disease, Intestinal epithelial barrier, Enteric nervous system, Tight junctions, Occludin, ZO-1

\section{Introduction}

The intestinal epithelium forms a regulated barrier, known as intestinal epithelial barrier (IEB), between the blood circulation and the contents of the intestinal lumen [1]. It prevents the passage of noxious contents while allowing the absorption and secretion of nutrients [1]. Penetration of this barrier occurs via two routes, either between epithelial cells via the paracellular pathway, or through epithelial cell via the transcellular pathway [1]. Among the most important structures of the intestinal barrier are the epithelial tight junctions (TJs) that connect adjacent enterocytes together to determine paracellular permeability through the lateral intercellular space [2]. They are formed by transmembrane proteins such as claudins and occludins connected to the actin cytoskeleton via high

\footnotetext{
* Correspondence: derkinderenp@yahoo.fr

${ }^{\dagger}$ Equal contributors

'Inserm U913, 1 rue Gaston Veil, Nantes F-44035, France

2University Nantes, Nantes F-44093, France

Full list of author information is available at the end of the article
}

molecular weight proteins called zona occludens (ZO-1, ZO-2 and 3) [2]. Increased permeability of the IEB along with changes in the expression levels of TJs proteins have been consistently reported in several digestive disorders such as inflammatory bowel disease [3,4] and irritable bowel syndrome $[5,6]$.

It has become evident over the last 20 years that PD is a gut disorder (reviewed in [7]). Gastrointestinal symptoms occur in almost every PD patient at some point and are among the most debilitating non-motor features of the disease [8]. These clinical data have been supported by post mortem studies that demonstrated the presence of Lewy bodies and neurites in the enteric neurons in nearly every case examined pathologically $[9,10]$. The German pathologist Heiko Braak suggested that the appearance of Lewy pathology in enteric neurons develop early in the course of disease, prior to the involvement of the central nervous system [11]. This led him to suggest that the gastrointestinal tract might be a portal of entry for a putative pathogen that would breach the IEB to induce the 
formation of Lewy bodies and neurites in the enteric neurons [11].

The high prevalence of gastrointestinal symptoms and pathology in PD and the possible derangement of gastrointestinal permeability in the pathogenesis of the disease prompted several groups to investigate IEB permeability in parkinsonian patients. The three studies, which have been carried out to date have all used absorption of sugar probes as a means to investigate non-invasively the paracellular permeability [12]. These studies, which have included a small number of patients, led to conflicting results. Two studies found a pattern of sugar absorption reminiscent of small intestine hyperpermeability in a subset of patients $[13,14]$ while the third one showed an increase in sucralose excretion without changes in the lactulose/ mannitol ratio, a pattern consistent with increased colonic permeability [15]. We therefore undertook the present research to analyze in more details the IEB in PD. To this end, a functional and structural characterization of the IEB was performed in colonic biopsies from PD patients.

\section{Materials and methods Subjects}

A total of 42 subjects participated in this study, 31 PD patients and 11 healthy controls. PD patients aged 43-74 years were recruited from the movement disorder clinic at Nantes University Hospital, France. Diagnosis of PD was made according to criteria provided by the United Kingdom Parkinson's Disease Survey Brain Bank [16]. Collected demographic data included gender, age at onset and disease duration, as well as age at colonoscopy. Complete drug history was obtained, and an approximation of the cumulative dose of L-dopa was made based on the equation developed by Parkkinen and collaborators [17]. Control subjects were healthy subjects who had a normal colonoscopy performed for colorectal cancer screening. All controls subjects underwent a detailed neurological examination to rule out PD symptoms and cognitive deficiency. Controls and PD patients were excluded if they suffered from irritable bowel syndrome and/or anorectal dysfunction. The study protocol was approved by the local Committee on Ethics and Human Research (Comité de Protection des Personnes Ouest VI) and registered on ClinicalTrials.gov (identifier NCT01748409). Written informed consent was obtained from each patient and from each normal volunteer according to the principles of Helsinki.

\section{Endoscopic procedure and colonic biopsies}

For each subject, nine biopsies were taken in the sigmoid/ descending colon during the course of a rectosigmoidoscopy for PD patients and during a colonoscopy for control subjects. Five biopsies were immersed in $4^{\circ} \mathrm{C}$ Hank's Balanced Salt Solution (Life Technologies, Saint Aubin, France): three of these biopsies were immediately processed for the assessment of para- and transcellular permeability in Ussing chambers while the two other biopsies were used for immunohistochemistry experiments. Two biopsies were stored at $-80^{\circ} \mathrm{C}$ in lysis buffer RA1 (Macherey-Nagel, Hoerdt, France) with 1\% (v/v) $\beta$-mercaptoethanol (Sigma, Saint Quentin Fallavier, France) for further analysis by immunoblotting. The two remaining biopsies were snap frozen in liquid nitrogen at the time of collection and kept at $-80^{\circ} \mathrm{C}$.

\section{Para- and transcellular permeability of colonic biopsies in Ussing chambers}

Three biopsies were mounted in Ussing chambers (World Precision Instruments; WPI, Hertfordshire, UK) exposing a surface of $0.011 \mathrm{~cm}^{2}$. Tissues were bathed on each side with $3 \mathrm{ml}$ of F12 supplemented Dulbecco's Modified Eagle medium (Invitrogen, France) containing $0.1 \%(\mathrm{v} / \mathrm{v})$ fetal bovine serum, $200 \mathrm{mM}$ Glutamine and $45 \mathrm{~g} / \mathrm{L}$ of $\mathrm{NaHCO}_{3}$. The medium was continuously oxygenated and maintained at $37^{\circ} \mathrm{C}$ by a gas flow $\left(95 \% \mathrm{O}_{2} / 5 \%\right.$ $\mathrm{CO}_{2}$ ). After a $30 \mathrm{~min}$ baseline period, $275 \mu \mathrm{L}$ of apical medium was replaced with $200 \mu \mathrm{L}$ of media containing $1 \mathrm{mg} / \mathrm{mL}$ of fluorescein-5,6-sulfonic acid (molecular weight: $400 \mathrm{Da}$ ) (Life Technologies) for a final concentration of $0.1 \mathrm{mg} / \mathrm{mL}$ to assess paracellular permeability. Seventy-five microliters of media with $10 \mathrm{mg} / \mathrm{mL}$ of Horse Radish Peroxydase (HRP) (Sigma) were also added to the basolateral chamber for a final concentration of $0.375 \mathrm{mg} / \mathrm{mL}$ to measure transcellular permeability in a subset of PD patients and control subjects. The fluorescence level of basolateral aliquots of $150 \mu \mathrm{l}$, reflecting paracellular transit from the luminal surface was measured every $30 \mathrm{~min}$ over a 3-hour period using a fluorimeter (Varioskan', ThermoFisher Scientific, Cillebon sur Yvette, France). HRP quantities in the basolateral chamber, reflecting transcellular transit from the apical surface, was measured using an enzymatic activity assay with 3,3,5,5'-tetramethylbenzidine reagent (BD Bioscience, Le Pont de Claix, France). Paracellular and transcellular permeabilities were determined by averaging the gradient of change in fluorescence intensity over time in the three biopsies that were analyzed per patient, using a linear regression fit model (GraphPad Prism 5, La Jolla, USA).

\section{Western blot}

For the analysis of ZO-1 expression, total proteins from the 2 biopsies stored in RA1 buffer were precipitated and prepared for Polyacrylamide Gel Electrophoresis (PAGE) using protein precipitator and resuspension buffer (Protein solving buffer and (tris(2-carboxyethyl) phosphine) TCEP reducing agent, PSB/TCEP) from NucleoSpin Triprep Kit (Macherey-Nagel, Hoerdt, France) according to the manufacturer's instructions. For experiments on the transmembrane protein occludin, the two 
dry frozen biopsies stored at $-80^{\circ} \mathrm{C}$ were lysed in UTC buffer (7 M Urea, $2 \mathrm{M}$ Thiourea and 4\% CHAPS) containing a protease inhibitor cocktail (Complete ${ }^{\mathrm{Tw}}$, Roche, Meylan, France) using the "Precellys 24" tissue homogenizer (Bertin technologies, Saint Quentin-en-Yvelines, France) and followed by sonication with "vibracell 75 186" device (Sonics, Newton CT, USA). Equal amounts of lysate were separated using the Invitrogen NuPage Novex 3-8\% Tris-Acetate Midi Protein Gels ${ }^{\mathrm{Tm}}$ for ZO-1 or NuPage Novex 4-12\% Bis-Tris MidiGels ${ }^{\mathrm{Tm}}$ for occludin before electrophoretic transfer to nitrocellulose membranes with the iBlot ${ }^{\text {tix }}$ Dry Blotting System also from Invitrogen. Membranes were processed for immunoblotting using rabbit polyclonal anti-ZO-1 (1:500, Life Technologies) and rabbit anti-occludin (1:250, Abcam, Paris, France) antibodies and the relevant immunoreactive bands were quantified as previously described [18].

\section{Microdissection and immunohistochemistry}

Microdissection was performed as previously described [19] in two out of the nine biopsies taken per patient. Each whole-mount preparation of submucosa obtained from a single biopsy was permeabilized for 3 hours in phosphate buffered saline (PBS)/ $\mathrm{NaN}_{3}$ containing $1 \%(\mathrm{v} / \mathrm{v})$ Triton $\mathrm{X}-100$ and $10 \%(\mathrm{v} / \mathrm{v})$ horse serum and then incubated with antibodies against phosphorylated alpha-synuclein (1:5000, WAKO, Osaka, Japan) and PGP9.5 (1:10,000; Ultraclone Limited, UK). Each whole-mount preparation of mucosa was treated for $24 \mathrm{~h}$ with Scale A2 solution composed of $4 \mathrm{M}$ urea, $10 \%(\mathrm{w} / \mathrm{v})$ glycerol and $0.1 \%(\mathrm{v} / \mathrm{v})$ Triton X100 [20] then incubated with rabbit polyclonal antibodies to ZO-1 (1:100, Life Technologies) and occludin (1:100, Abcam). Suitable secondary antibodies conjugated to Alexa Fluor 488 and 594 were used (Invitrogen, CergyPontoise, France). Following incubation with the secondary antibodies, the mucosa samples were treated for 10 minutes with a solution of $0.3 \%(\mathrm{w} / \mathrm{v})$ of Sudan Black B powder (Sigma) dissolved in 70\% (v/v) ethanol, then washed extensively with PBS. Whole specimen of submucosa and mucosa were viewed under an Axio Zoom.V16 stereomicroscope (Zeiss, Marly Le Roi, France). All samples were deidentified and studied in a blinded manner. For the analysis of ZO-1 and occludin immunofluorescence, the percentage of morphologically normal crypts per biopsy was calculated and the following classification was used: 'normal', more than $2 / 3$ of morphologically normal crypts; 'mild disruption': between $1 / 3$ and $2 / 3$ of morphologically normal crypts; 'disrupted': less than $1 / 3$ of morphologically normal crypts.

\section{Statistics}

All data are given as the mean \pm standard error of the mean (SEM). For comparisons of means between groups, a Mann-Whitney test was performed. Differences were deemed statistically significant if $\mathrm{p}<0.05$.

\section{Results}

A total of 31 PD patients and 11 controls were included. Table 1 shows the main clinical and demographic features of all patients. Age and sex did not differ significantly between PD patients and control subjects (mean age was $64.2 \pm 2.1$ for $\mathrm{PD}$ and $60.6 \pm 1.4$ for controls, $\mathrm{p}=0.25 ; 22 / 31$ male in PD group and $6 / 11$ male in control group, $\mathrm{p}=0.13$ ).

\section{Para- and transcellular permeability are unaffected in PD}

In a first set of experiments, we evaluated whether IEB is functionally altered in PD patients. The para- and

Table 1 Main clinical characteristics of PD patients

\begin{tabular}{|c|c|c|c|c|}
\hline & Age & Sex & $\begin{array}{l}\text { Disease duration } \\
\text { (years) }\end{array}$ & $\begin{array}{l}\text { Cumulative lifetime } \\
\text { dose of L-dopa (mg) }\end{array}$ \\
\hline 1 & 70 & $F$ & 2 & 0 \\
\hline 2 & 64 & $M$ & NK & NK \\
\hline 3 & 70 & $M$ & NK & NK \\
\hline 4 & 66 & $M$ & 23 & 66111000 \\
\hline 5 & 47 & $F$ & 2 & 0 \\
\hline 6 & 62 & $M$ & 4 & 3823375 \\
\hline 7 & 74 & $M$ & 13 & 1505625 \\
\hline 8 & 53 & $M$ & 4 & 2372500 \\
\hline 9 & 69 & $M$ & 2.5 & 273750 \\
\hline 10 & 43 & $F$ & 11 & 508050 \\
\hline 11 & 67 & $M$ & 2.3 & 0 \\
\hline 12 & 58 & $M$ & 7 & 2135250 \\
\hline 13 & 58 & $M$ & 9 & 1533000 \\
\hline 14 & 72 & $F$ & 14 & 1442662.5 \\
\hline 15 & 64 & $M$ & 4 & 492750 \\
\hline 16 & 64 & $M$ & 10 & 355875 \\
\hline 17 & 56 & $M$ & NK & NK \\
\hline 18 & 66 & $M$ & 4.6 & 0 \\
\hline 19 & 62 & $M$ & 26 & 1560375 \\
\hline 20 & 70 & $F$ & 2.2 & 219000 \\
\hline 21 & 57 & $M$ & 1.8 & 0 \\
\hline 22 & 58 & $M$ & 13 & 2007500 \\
\hline 23 & 50 & $M$ & 8 & 1286625 \\
\hline 24 & 69 & $F$ & 28 & 3759500 \\
\hline 25 & 62 & $M$ & 8 & 511000 \\
\hline 26 & 56 & $F$ & 8 & 1241000 \\
\hline 27 & 46 & $M$ & 10 & 1213625 \\
\hline 28 & 56 & $F$ & 8.4 & 866875 \\
\hline 29 & 58 & $F$ & 4.8 & 447125 \\
\hline 30 & 54 & $M$ & 24 & 3085423 \\
\hline 31 & 58 & $M$ & 10 & 3374130 \\
\hline
\end{tabular}

NK: not known. 
transcellular permeability of colonic biopsies were measured in Ussing chambers in both PD patients and control subjects using sulfonic acid and HRP, respectively. No difference in the sulfonic acid flux was observed between PD patients and control subjects $(\mathrm{n}=31$ and 11 , respectively; $\mathrm{p}=0.65$ ) (Figure $1 \mathrm{~A}$ ). HRP flux was also comparable between PD subjects and healthy controls $(\mathrm{n}=21$ and 9 , respectively; $\mathrm{p}=0.39)$ (Figure $1 \mathrm{~B})$. Although not statistically different from controls, the sulfonic acid and HRP flux values were heterogeneous between PD patients (Figure 1A and B). We thus investigated if the main clinical features of the disease had any influence on IEB

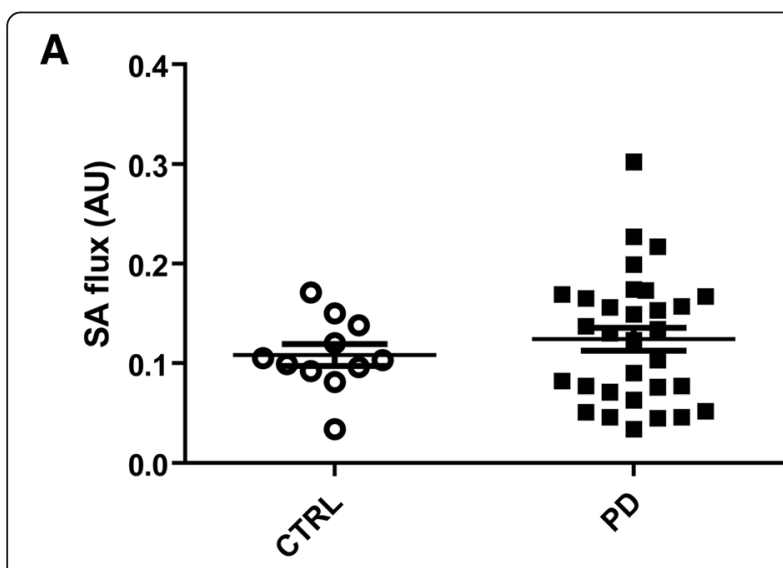

B

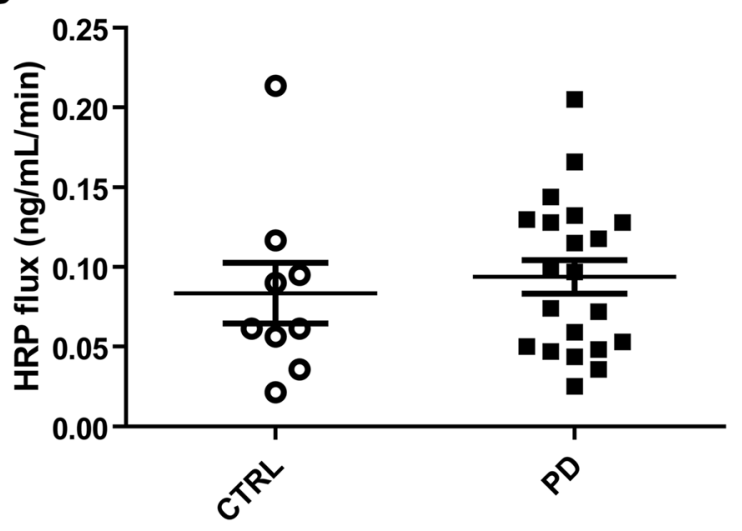

Figure 1 Comparison of para- and transcellular permeability in PD patients and healthy controls. (A) For the evaluation of paracellular permeability, the flux of sulfonic acid (SA flux) was measured in colonic biopsies mounted in Ussing chambers, expressed in arbitrary units (AU), in PD patients $(n=31)$ and controls (CTRL, $n=11)$. No significant changes were observed between the two groups $(p=0.65)$. (B) For the evaluation of paracellular permeability, the flux of horseradish peroxidase (HRP flux) was measured in colonic biopsies mounted in Ussing chambers, expressed in $\mathrm{ng} / \mathrm{mL} / \mathrm{min}$, in PD patients $(n=21)$ and controls ( $C T R L, n=9)$. No significant changes were observed between the two groups $(p=0.39)$. permeability. We did not observe any correlation between age, disease duration or lifetime cumulative dose of LDOPA and the values of sulfonic acid or HRP flux (Table 2).

There is a growing body of evidence supporting a key role for submucosal enteric neurons in the regulation of IEB functions [21-23]. This prompted us to study if the flux values of sulfonic acid and HRP were related to the presence of Lewy bodies and Lewy neurites in the submucosa. To this end, two biopsies per patient were immunohistochemically assessed for the presence of Lewy pathology using antibodies against phosphorylated alpha-synuclein and PGP9.5 (Figure 2A and B). A biopsy was deemed positive when containing at least one inclusion immunoreactive for both phosphorylated alphasynuclein and PGP9.5 (Figure 2C). A patient was noted as positive when at least one of the two biopsies displayed inclusion(s). In accordance with our previous reports [24,25], the intraneuronal inclusions found in the submucosal plexus were chiefly observed in the neuronal processes and thus reminiscent of Lewy neurites (Figure 2A-C). Twenty-three out of 31 PD patients were positive for phosphorylated alpha-synuclein inclusions. All control subjects were devoid of inclusions. The values of sulfonic acid and HRP flux were not different between PD patients with or without inclusions (Figure 2D and E).

\section{Expression of the tight junction protein occludin is decreased in PD}

We next investigated whether structural changes in the IEB occur in PD. The expression levels of the TJs proteins ZO-1 and occludin were analyzed by Western blot in colonic biopsies from PD subjects and healthy controls. A significant decrease in the expression of occludin was observed in colonic samples of PD patients as compared to controls (Figure 3A and B). A doublet band of approximately $220 \mathrm{kDa}$ was observed on Western blot with antibodies against ZO-1 (Figure 3A). As previously shown, these two bands most likely represent the two ZO-1 isoforms [26,27]. By contrast to occludin, no change in ZO-1 expression levels was observed in PD whether the two bands were quantified together (Figure 3C) or separately (data not shown).

Table 2 Spearman's correlation with sulfonic acid (SA) and HRP permeability in PD patients

\begin{tabular}{llll}
\hline & Age & $\begin{array}{l}\text { Disease } \\
\text { duration }\end{array}$ & $\begin{array}{l}\text { Cumulative lifetime } \\
\text { dose of L-dopa }\end{array}$ \\
\hline SA & $p=0.090$ & $p=0.788$ & $p=0.830$ \\
HRP & $r=0.303$ & $r=0.052$ & $r=-0.046$ \\
& $p=0.165$ & $p=0.469$ & $p=0.754$ \\
& $r=0.323$ & $r=0.172$ & $r=0.082$ \\
\hline
\end{tabular}



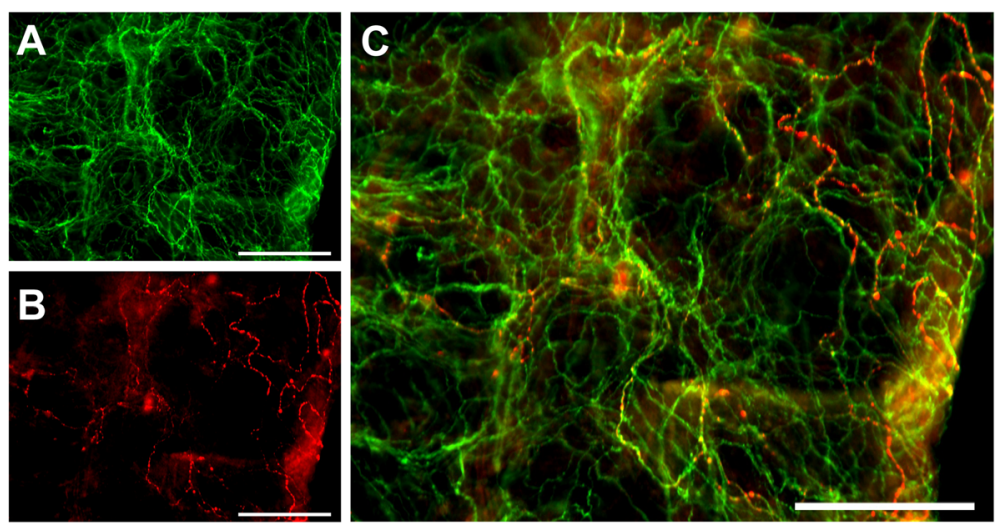

D

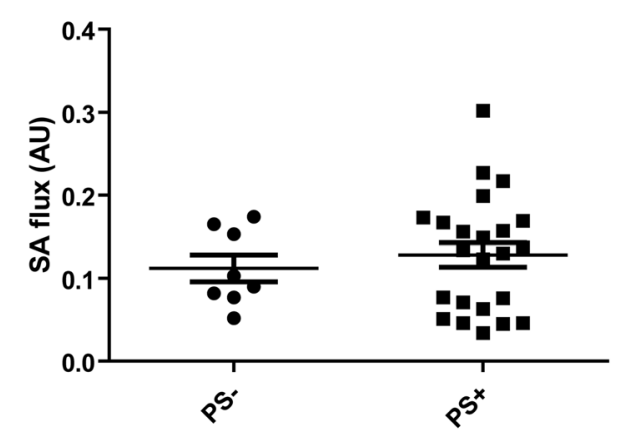

$\mathbf{E}$

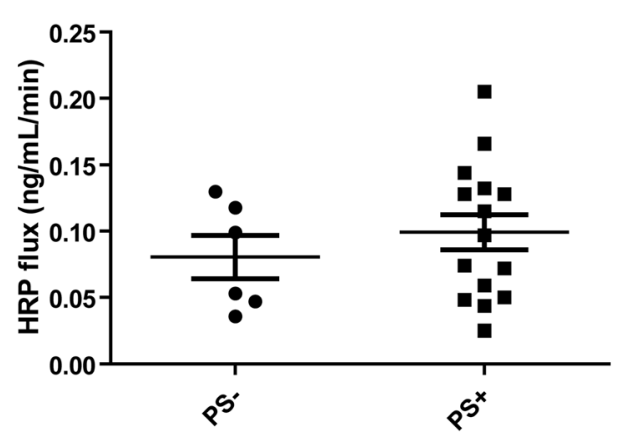

Figure 2 Sulfonic acid and HRP flux in PD in patients with and without enteric Lewy pathology. Representative photomicrograph depicting multiple Lewy neurites in a whole-mount of submucosa immunoreactive for PGP9.5 (A) that was also positive for phosphorylated alpha-synuclein (B and C) Scale bar: $200 \mu \mathrm{m}$. (D) The values of sulfonic acid flux was not different between PD patients with (PS+) or without (PS-) phospho-synuclein immunoreactive neurites $(p=0.93)$. (E) The values of HRP flux was not different between PD patients with (PS+) or without (PS-) phospho-synuclein immunoreactive neurites $(p=0.46)$.

\section{Cellular distribution of the TJs proteins is altered in PD} The cellular distribution of occludin and ZO-1 was further investigated by immunofluorescence in 8 controls and 31 PD subjects. Samples from 3 controls were excluded because the mucosa was too small and/or too damaged to allow a reliable analysis of the TJs morphology. A mean of 96.4 crypts per biopsy were analyzed. We observed differences in the cellular distribution of both ZO-1 and occludin between PD patients and controls (Figure 4). A normal and typical reticular pattern of occludin and ZO-1 staining was observed in the colonic samples of 6 out of 8 controls (Figure 4A and C, Additional file 1) and in only 9/31 PD patients (Figure 4B and $\mathrm{D}$, Additional file 1). TJs morphology was disrupted and irregularly distributed in the mucosa of 1 out of 8 controls (Figure 4A and C, Additional file 1) and in 14/ 31 PD patients (Figure $4 \mathrm{~B}$ and D, Additional file 1). An occasional and mild disruption of TJs morphology was 


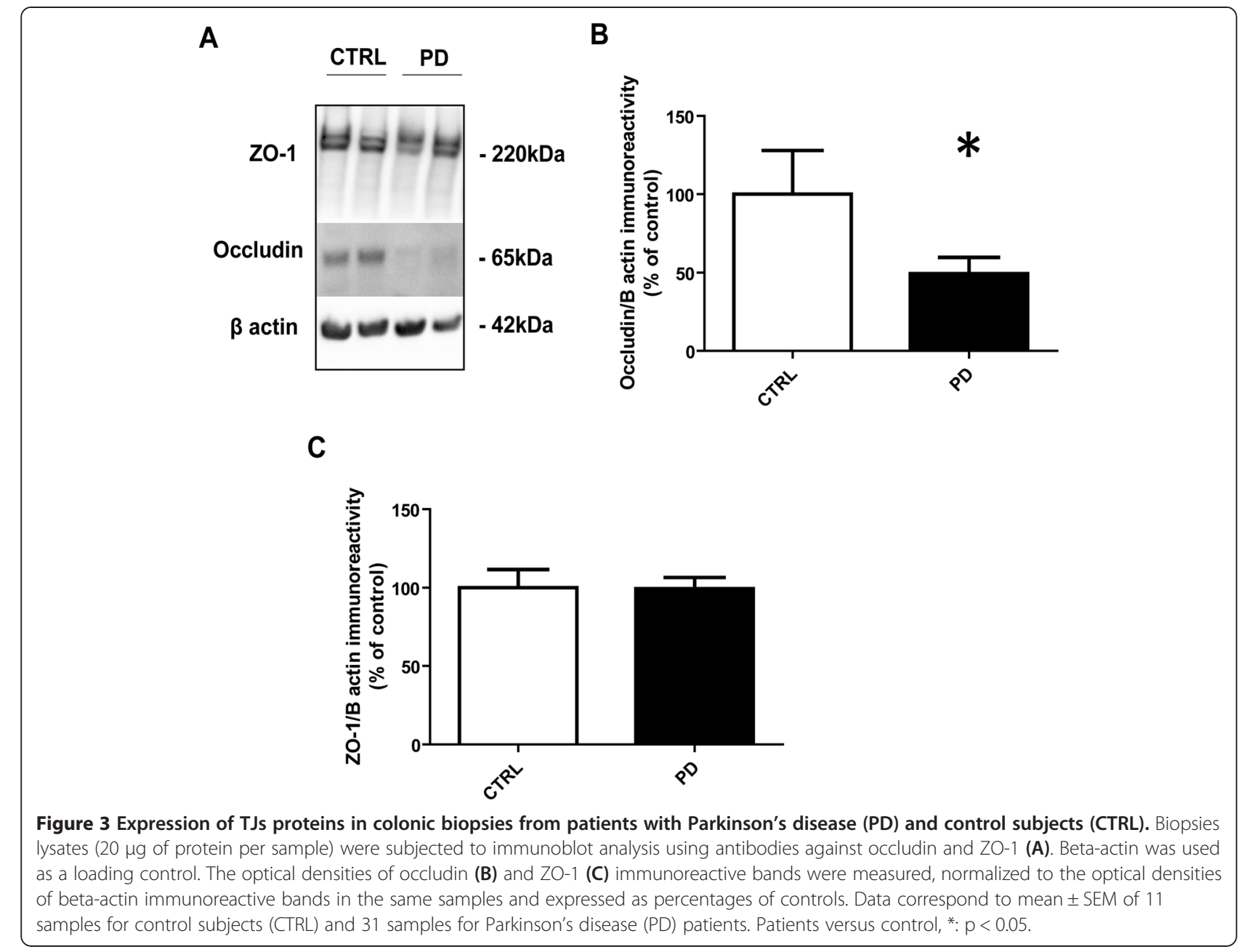

B

observed in the remaining control subject and in 8/31 PD samples (Figure 4 and Additional file 1). An increased staining of occludin in the cytoplasm of colonic enterocytes, suggestive of protein internalization, was observed in PD samples as opposed to the healthy group where occludin was mostly located in the TJs (Figure 4C and D). Worthy of note was the presence of moderate to severe TJs disorganization in the 5 patients who had never received levodopa, suggesting that the altered TJs morphology was not related to chronic levodopa intake (Additional file 2 and Table 1).

\section{Discussion}

The TJs are intercellular protein complex located at the apical portions of the lateral membranes of epithelial cells which play a key role in the regulation of IEB paracellular permeability. They are composed of transmembrane proteins, such as claudins and occludin and a wide spectrum of cytosolic proteins among which is ZO-1 [1]. By showing a decrease in occludin expression along with TJs disorganization, our study is the first to provide evidence that the IEB is structurally altered in PD. Previous studies have shown intestinal tissue expression and distribution of occludin to be markedly decreased in patients with intestinal permeability disorders, including inflammatory bowel disease [28] and irritable bowel syndrome [6]. Recent data from genetic and epidemiological studies provided support for an association between diseases of the gastrointestinal tract and the susceptibility to developing PD. The CARD15 gene known to be associated with Crohn's disease is over-represented in patients with PD [29]; vice versa, the Leucine-rich repeat kinase 2 (LRRK2) gene, a causative PD mutation, was recently identified as a major susceptibility gene for Crohn's disease by genome-wide association studies [30]. Moreover, patients with irritable bowel syndrome are almost $50 \%$ more likely to develop PD than people who are free of this gastrointestinal disorder [31]. Our results show that the two disorders also share similarities at the molecular levels further supporting the assumption that irritable bowel syndrome may actually belong to early signs of gastrointestinal involvement in PD [32]. They also support 


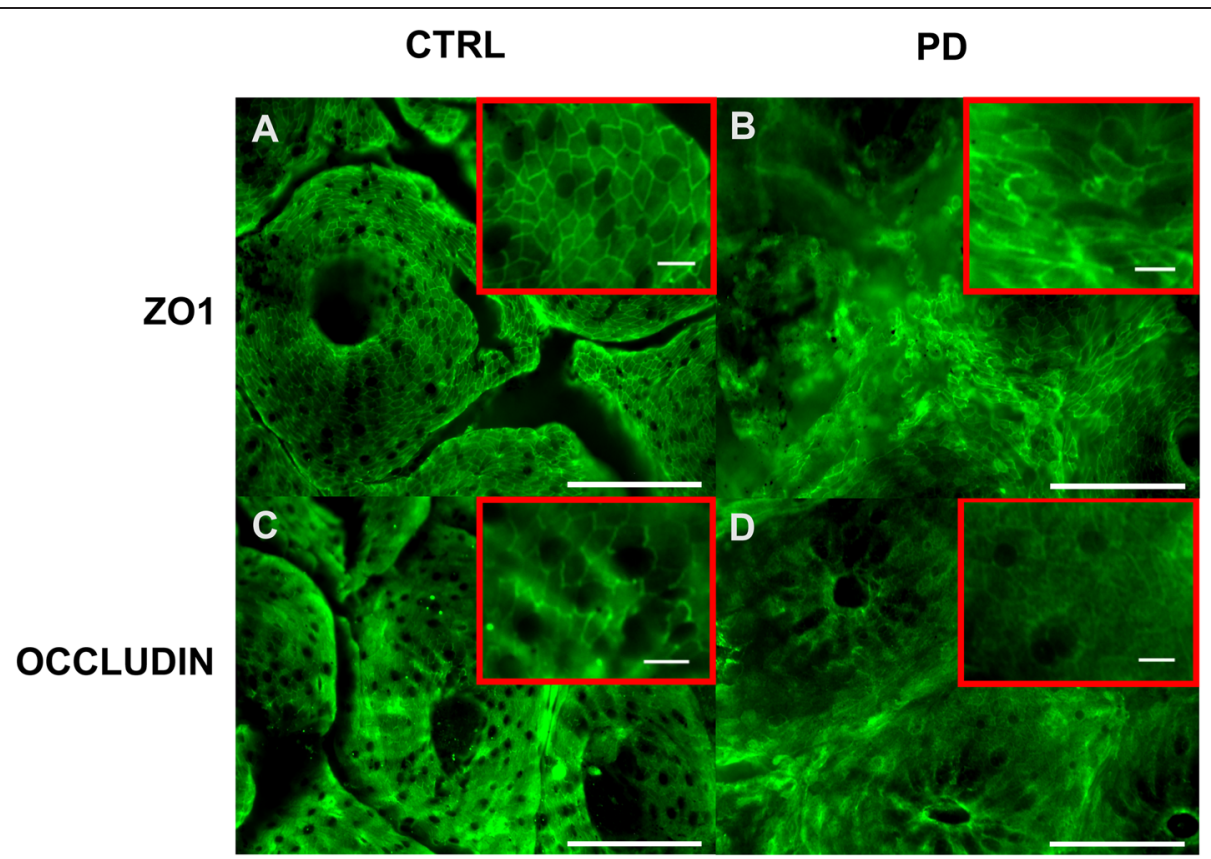

Figure 4 Localization of TJs proteins in the colonic mucosa of healthy controls (CTRL) and patients with Parkinson's disease (PD).

Representative photomicrographs of the colonic mucosa labeled with antibodies against ZO-1 (A, B) and occludin (C, D) in the colonic mucosa of control and PD patients; scale bar: $100 \mu \mathrm{m}$. High-magnification image of each area marked by red square; scale bar: $10 \mu \mathrm{m}$.

the hypothesis that the brain gut-axis might be critically involved in the pathophysiology of both disorders [7,33].

Studies of intestinal permeability in humans have mainly been carried out with in vivo techniques, usually with oral ingestion of various sugar probes and measurement of urinary excretion [12]. To date, the three studies that attempted to evaluate intestinal permeability in PD using this technique have provided only preliminary and conflicting results. Two of these studies focused on the lactulose/mannitol ratio, which evaluate small intestinal permeability. As a group, the 15 PD patients studied by Davies and collaborators had a significant increase in the lactulose/mannitol ratio when compared to age and sex matched controls, but individual results in both groups were highly overlapping [14]. Salat-Foix et al. showed that the lactulose/mannitol ratio was only marginally higher in 3 out of 12 PD patients [13]. In addition to lactulose/mannitol ratio, Forsyth et al. also used sucralose absorption for the assessment of colon permeability in 9 PD patients and 10 controls. They did not observe any difference in the lactulose/mannitol ratio between the two groups but found a significantly greater permeability to sucralose in PD subjects [15]. The inconsistent results on intestinal permeability in PD obtained with sugar probes prompted us to measure IEB permeability by another technique, namely Ussing chambers, in a larger sample size. Although less commonly used than sugar absorption, the Ussing chambers has proven to be a reliable and effective tool to measure IEB permeability of gastrointestinal biopsies either paracellularly or transcellularly over a 3 hour period [34]. Using this approach, we showed that there were no significant differences in para- and transcellular permeabilities between PD subjects and controls. Nevertheless, the values of paracellular permeability as assessed by the sulfonic acid flux in Ussing chamber were highly heterogeneous between PD patients, some displaying a level comparable to controls while others had a more than a 2.5 fold increase in sulfonic acid flux. These data suggest that increased colonic permeability may be a feature for a subset of PD patients, as already reported when sugar probes were used [15]. The factors responsible for this heterogeneity still remain to be determined, as we did not observe any correlation between age, cumulative dose of L-Dopa disease duration and the severity of altered permeability.

On the surface, our results may seem contradictory, as the decreased expression of occludin observed in PD patients was not accompanied by changes in paracellular permeability. Occludin is a tetraspan protein with two extracellular loops, which homophilically interact with the adjacent cells [2]. Its role on IEB has been debated since its initial discovery in 1993 [35]. Initial studies strongly suggested that occludin was not required for the TJs formation or the maintenance of barrier function as occludin knockout mice lacked any noticeable defect in intestinal TJs morphology or barrier function [36]. This has been recently challenged in an elegant study 
published by Al-Sadi et al. [37]. The purpose of their research was to better delineate the involvement of occludin in IEB by studying the transepithelial flux of various-sized probes after knocking down occludin both in vitro and in vivo. They showed that the occludin knock down caused a marked increase in the flux rates of macromolecules above $5 \mathrm{kDa}$ such as inulin and dextran but had only modest effect on flux of smaller-sized probes under $200 \mathrm{Da}$ such as mannitol and urea [36]. Fluorescein-5,6-sulfonic acid, which was used for the assessment of paracellular permeability in our study has a molecular weight of $400 \mathrm{Da}$, likely to be too small for detecting defects in IEB permeability induced by a mere down regulation of occludin. This may explain the lack of significant changes in IEB permeability observed in PD patients in our study in spite of the occurrence of structural changes.

The question arises as to what might be the clinical relevance of our experimental findings. A current theory, the so-called Braak's theory, assumes that PD originates in the gastrointestinal tract [11]. Braak and co-workers suggested that the appearance of Lewy pathology occurs in the earliest stage of PD in both the enteric nervous system and the dorsal motor nucleus of the vagus $[11,38]$. This led Braak to postulate that a pathogen may breach the IEB to trigger Lewy pathology in the terminal axons of the enteric neurons, further spreading to the central nervous system via the vagal preganglionic innervation of the gut $[11,39]$. In light of these considerations, our results demonstrating altered intestinal TJs structure in PD gain in importance as the down regulation of occludin may favor the entry of a putative pathogen. This must be however balanced, as the stomach in contrast to the colon appears to be the most suitable target for the pathologic insult to occur in Braak's scenario. Several studies have indeed described that Lewy pathology is distributed following a rostro-caudal gradient in $\mathrm{PD}$, with the lower esophagus and stomach having the greatest involvement and the colon and rectum the lowest $[9,10]$, a distribution that parallels the vagal innervation of the gastrointestinal tract [40]. Further studies are therefore warranted to analyze the mucosal barrier permeability and morphology in gastric and duodenal samples from PD patients.

\section{Conclusions}

In conclusion, we provide evidence for the first time that morphological changes in the IEB occur in PD patients. Our results further reinforce the possible role of the gastrointestinal tract in the pathophysiology of $\mathrm{PD}$. Further work is needed to determine if occludin down regulation in the gut might facilitate the spreading of PD pathology in the enteric nervous system and in the brain.

\section{Additional files}

Additional file 1: Percentage of morphologically normal crypts in the colonic mucosa of healthy controls (CTRL) and patients with Parkinson's disease (PD).

Additional file 2: Localization of TJs proteins in the colonic mucosa of the 5 with Parkinson's disease (PD) who had never received levodopa. Scale bar: $100 \mu \mathrm{m}$.

\section{Competing interests}

The authors declare that they have no competing interests.

\section{Acknowledgements}

This work was supported by a grant from the Michael J. Fox Foundation for Parkinson's Research (Rapid Response Innovation Award 2013) and France Parkinson to PD. LLV is supported by a grant from Nantes University Hospital (Appel d'offre interne 2012, Grant number RC12_0264) and France Parkinson. TC is supported by a grant from centre d'entraide et de coordination des associations de parkinsoniens (CECAP).

\section{Author details}

${ }^{1}$ Inserm U913, 1 rue Gaston Veil, Nantes F-44035, France. ²University Nantes, Nantes F-44093, France. ${ }^{3} \mathrm{CHU}$ Nantes, Institut des Maladies de I'Appareil Digestif, Nantes F-44093, France. ${ }^{4}$ Inserm, ClC-04, Nantes F-44093, France. ${ }^{5} \mathrm{CHU}$ Nantes, Service d'Anatomie Pathologique, Nantes F-44093, France. ${ }^{6}$ Inserm, UMR957, Nantes F-44093, France. ${ }^{7} \mathrm{CHU}$ Nantes, Department of Neurology, Nantes F-44093, France.

Received: 8 February 2015 Accepted: 10 February 2015

Published online: 10 March 2015

\section{References}

1. Marchiando AM, Graham WV, Turner JR (2010) Epithelial barriers in homeostasis and disease. Annu Rev Pathol 5:119-44

2. Suzuki T (2013) Regulation of intestinal epithelial permeability by tight junctions. Cell Mol Life Sci 70:631-59

3. Peeters M, Ghoos Y, Maes B, Hiele M, Geboes K, Vantrappen G et al (1994) Increased permeability of macroscopically normal small bowel in Crohn's disease. Dig Dis Sci 39:2170-6

4. Katz KD, Hollander D, Vadheim CM, McElree C, Delahunty T, Dadufalza VD et al (1989) Intestinal permeability in patients with Crohn's disease and their healthy relatives. Gastroenterology 97:927-31

5. Piche T, Barbara G, Aubert P, Bruley des Varannes S, Dainese R, Nano JL et al (2009) Impaired intestinal barrier integrity in the colon of patients with irritable bowel syndrome: involvement of soluble mediators. Gut 58:196-201

6. Bertiaux-Vandaële N, Youmba SB, Belmonte L, Lecleire S, Antonietti M, Gourcerol G et al (2011) The expression and the cellular distribution of the tight junction proteins are altered in irritable bowel syndrome patients with differences according to the disease subtype. Am J Gastroenterol 106:2165-73

7. Derkinderen P, Rouaud T, Lebouvier T, Bruley des Varannes S, Neunlist M, De Giorgio R (2011) Parkinson disease: the enteric nervous system spills its guts. Neurology 77:1761-7

8. Cloud LJ, Greene JG (2011) Gastrointestinal features of Parkinson's disease. Curr Neurol Neurosci Rep 11:379-84

9. Wakabayashi K, Takahashi H, Takeda S, Ohama E, Ikuta F (1988) Parkinson's disease: the presence of Lewy bodies in Auerbach's and Meissner's plexuses. Acta neuropathologica 76:217-21

10. Beach TG, Adler CH, Sue LI, Vedders L, Lue L, White lii CL et al (2009) Multi-organ distribution of phosphorylated alpha-synuclein histopathology in subjects with Lewy body disorders. Acta neuropathologica 119:689-702

11. Braak H, de Vos RA, Bohl J, Del Tredici K (2006) Gastric alpha-synuclein immunoreactive inclusions in Meissner's and Auerbach's plexuses in cases staged for Parkinson's disease-related brain pathology. Neurosci Lett 396:67-72

12. Hollander D (1999) Intestinal permeability, leaky gut, and intestinal disorders. Curr Gastroenterol Rep 1:410-6

13. Salat-Foix D, Tran K, Ranawaya R, Meddings J, Suchowersky O (2012) Increased intestinal permeability and Parkinson disease patients: chicken or egg? Can J Neurol Sci 39:185-8 
14. Davies KN, King D, Billington D, Barrett JA (1996) Intestinal permeability and orocaecal transit time in elderly patients with Parkinson's disease. Postgrad Med J 72:164-7

15. Forsyth CB, Shannon KM, Kordower JH, Voigt RM, Shaikh M, Jaglin JA et al (2011) Increased intestinal permeability correlates with sigmoid mucosa alpha-synuclein staining and endotoxin exposure markers in early Parkinson's disease. PLoS One 6:e28032

16. Hughes AJ, Daniel SE, Lees AJ (2001) Improved accuracy of clinical diagnosis of Lewy body Parkinson's disease. Neurology 57:1497-9

17. Parkkinen L, O'Sullivan SS, Kuoppamaki M, Collins C, Kallis C, Holton JL et al (2011) Does levodopa accelerate the pathologic process in Parkinson disease brain? Neurology 77:1420-6

18. Clairembault T, Kamphuis W, Leclair-Visonneau L, Rolli-Derkinderen M, Coron E, Neunlist M et al. Enteric GFAP expression and phosphorylation in Parkinson's disease. J Neurochem 2014; doi:10.1111/jnc.12742.

19. Lebouvier T, Coron E, Chaumette T, Paillusson S, Bruley des Varannes S, Neunlist M et al (2010) Routine colonic biopsies as a new tool to study the enteric nervous system in living patients. Neurogastroenterol Motil 22:e11-4

20. Hama H, Kurokawa H, Kawano H, Ando R, Shimogori T, Noda H et al (2011) Scale: a chemical approach for fluorescence imaging and reconstruction of transparent mouse brain. Nat Neurosci 14:1481-8

21. Neunlist M, Aubert P, Toquet C, Oreshkova T, Barouk J, Lehur PA et al (2003) Changes in chemical coding of myenteric neurones in ulcerative colitis. Gut 52:84-90

22. Toumi F, Neunlist M, Cassagnau E, Parois S, Laboisse CL, Galmiche JP et al (2003) Human submucosal neurones regulate intestinal epithelial cell proliferation: evidence from a novel co-culture model. Neurogastroenterol Motil 15:239-42

23. Cameron HL, Perdue MH (2007) Muscarinic acetylcholine receptor activation increases transcellular transport of macromolecules across mouse and human intestinal epithelium in vitro. Neurogastroenterol Motil 19:47-56

24. Lebouvier $T$, Neunlist M, Bruley des Varannes $S$, Coron E, Drouard A, N'Guyen JM et al (2010) Colonic biopsies to assess the neuropathology of Parkinson's disease and its relationship with symptoms. PLoS One 5:e12728

25. Pouclet H, Lebouvier T, Coron E, Des Varannes SB, Neunlist M, Derkinderen P (2012) A comparison between rectal and colonic biopsies to detect Lewy pathology in Parkinson's disease. Neurobiol Dis 45:305-9

26. Ciana A, Meier K, Daum N, Gerbes S, Veith M, Lehr CM et al (2010) A dynamic ratio of the alpha + and alpha- isoforms of the tight junction protein ZO-1 is characteristic of Caco-2 cells and correlates with their degree of differentiation. Cell Biol Int 34:669-78

27. Willott E, Balda MS, Heintzelman M, Jameson B, Anderson JM (1992) Localization and differential expression of two isoforms of the tight junction protein ZO-1. Am J Physiol 262:C1119-24

28. Gassler N, Rohr C, Schneider A, Kartenbeck J, Bach A, Obermüller N et al (2001) Inflammatory bowel disease is associated with changes of enterocytic junctions. Am J Physiol Gastrointest Liver Physiol 281:G216-28

29. Bialecka M, Kurzawski M, Klodowska-Duda G, Opala G, Juzwiak S, Kurzawski $\mathrm{G}$ et al (2007) CARD15 variants in patients with sporadic Parkinson's disease. Neurosci Res 57:473-6

30. Barrett JC, Hansoul S, Nicolae DL, Cho JH, Duerr RH, Rioux JD et al (2008) Genome-wide association defines more than 30 distinct susceptibility loci for Crohn's disease. Nat Genet 40:955-62

31. Lai S-W, Liao K-F, Lin C-L, Sung F-C (2014) Irritable bowel syndrome correlates with increased risk of Parkinson's disease in Taiwan. Eur J Epidemiol 29:57-62, doi:10.1007/s10654-014-9878-3

32. Cersosimo MG, Raina GB, Pecci C, Pellene A, Calandra CR, Gutiérrez C et al (2013) Gastrointestinal manifestations in Parkinson's disease: prevalence and occurrence before motor symptoms. J Neurol 260:1332-8

33. Coss-Adame E, Rao SSC (2014) Brain and gut interactions in irritable bowel syndrome: new paradigms and new understandings. Curr Gastroenterol Rep 16:379

34. Wallon C, Braaf Y, Wolving M, Wolving M, Olaison G, Söderholm JD (2005) Endoscopic biopsies in Ussing chambers evaluated for studies of macromolecular permeability in the human colon. Scand J Gastroenterol 40:586-95

35. Furuse M, Hirase T, Itoh M, Nagafuchi A, Yonemura S, Tsukita S et al (1993) Occludin: a novel integral membrane protein localizing at tight junctions. J Cell Biol 123:1777-88
36. Saitou M, Furuse M, Sasaki H, Schulzke JD, Fromm M, Takano H et al (2000) Complex phenotype of mice lacking occludin, a component of tight junction strands. Mol Biol Cell 11:4131-42

37. Al-Sadi R, Khatib K, Guo S, Ye D, Youssef M, Ma T (2011) Occludin regulates macromolecule flux across the intestinal epithelial tight junction barrier. Am J Physiol Gastrointest Liver Physiol 300:G1054-64

38. Braak H, Del Tredici K, Rub U, de Vos RA, Jansen Steur EN, Braak E (2003) Staging of brain pathology related to sporadic Parkinson's disease. Neurobiol Aging 24:197-211

39. Braak H, Rub U, Gai WP, Del Tredici K (2003) Idiopathic Parkinson's disease: possible routes by which vulnerable neuronal types may be subject to neuroinvasion by an unknown pathogen. J Neural Transm 110:517-36

40. Hopkins DA, Bieger D, deVente J, Steinbusch WM (1996) Vagal efferent projections: viscerotopy, neurochemistry and effects of vagotomy. Prog Brain Res 107:79-96

\section{Submit your next manuscript to BioMed Central and take full advantage of:}

- Convenient online submission

- Thorough peer review

- No space constraints or color figure charges

- Immediate publication on acceptance

- Inclusion in PubMed, CAS, Scopus and Google Scholar

- Research which is freely available for redistribution 